SESSION IX. SUMMARY - Chair: Karel A. van der Hucht 


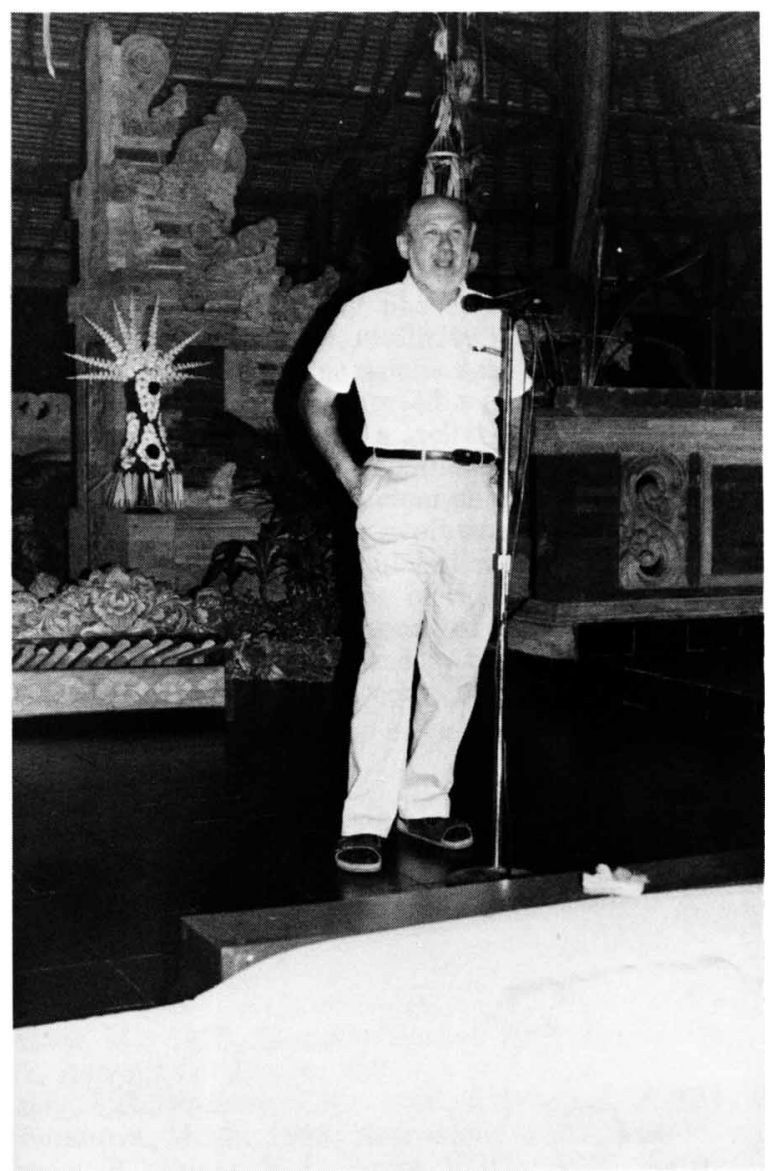

Peter Conti 


\title{
SUMMARY OF SYMPOSIUM
}

\author{
PETER S. CONTI \\ Joint Institute for Laboratory Astrophysics \\ University of Colorado, Boulder, CO 80309-0440, USA
}

\begin{abstract}
"It is a significant fact that the emission and absorption lines of Wolf-Rayet spectra cannot be regarded as being formed in one body of gas at one representative temperature and pressure" (Underhill 1968)
\end{abstract}

"I think it fair to say that the demonstration of ... diversity has brought the WolfRayet stars from being a curiosity to being an important part of the 'main stream' evolution of all massive stars" (Smith 1982)

It is not easy to put together, from the past four and one half days and into the space of about a half an hour, a fully concise summary of what has been presented here at our meeting. What follows must necessarily be a highly personalized view of these proceedings. My report is colored not only by my pre-conceptions but also by my own active participation in research on Wolf-Rayet stars and my singular interpretation of what is important, new, and newsworthy. I hope you will bear with me if not every contribution was singled out, and you will accept my own rendering of the various research results presented here. With a view to save time (and space) and to avoid potential hard feelings, I will not quote the authors of the contributions which may be readily found in any case in these proceedings.

The quotations that lead off this Summary come from the only two astronomers at this meeting who have also attended both previous IAU Symposia and the Boulder mini-symposium on the topic of Wolf-Rayet (W-R) stars. Their well known "antipodal" attitudes represent dramatically different interpretations of the nature of the spectra, and ultimately the understanding of, W-R stars. The Boulder meeting in 1968, coming some hundred years after the discovery of such stars, may be said to have begun the modern era of work in this field. You have already heard at the after dinner talk earlier in the week a summary of the initial "historical" period by Len Kuhi. In this paper, I shall briefly review the major themes of the previous meetings, so as to put into context the main results of this one. It must be remembered that considerable research and publication has gone on in between these Symposia but in briefly looking back at them we may obtain a flavor of the work that has ensued.

The Boulder mini-symposium (Gebbie and Thomas 1968) contains the modern definition of the W-R phenomena, in terms of a number of spectroscopic (i.e. observed) properties of the class, both those 
of Population I and certain central stars of planetary nebulae. There was much discussion of the newly emerging quantitative empirical classification of the WN and WC and their numerical subtypes. The overall composition, still very poorly understood, was argued about incessantly. The first indications of the anomalous galactic distribution of W-R subtypes was presented and tentative interpretations offered. Only primitive atmospheric models existed which were little more than "handwaving" in complexity. Considerable "yelling" about such models must have occurred at this meeting as may be inferred from the tone and nature of the proceedings. I was sorry to have missed this conference as my own interests in hot luminous stars were just beginning to stir at about this time. The publication time for the proceedings was under six months, a goal often promised but rarely honored.

In Buenos Aires in 1971 (Bappu and Sahade 1973) a major thrust of Symposium \#49 was devoted to binary evolution. At the time, nearly all of us thought that ALL W-R stars were double and their anomalous spectroscopic behavior would be able to be understood in terms of "mass exchange" and "stripping" of the initial primary in close binary systems. The first clear demonstration that the ionization decreased outwards (at least in WC stars) was shown. The first UV data (OAO) and the first radio data were presented. "Single point" atmospheric models were introduced: they gave some confidence to our perception of the anomalous composition of W-R stars. A comparison with optical emission line strengths was in its infancy.

A decade later, in Cozumel in 1981 at IAU Symposium \#99 (De Loore and Willis 1982), a major thrust was the discussion of "winds" in W-R stars. This term had been used before only sparingly; now it became a way of conceptualizing the spectra of W-R stars. Massive star evolution scenarios were calculated for single stars; the realization of the importance of mass loss and mixing had occurred in the recent past. The notion was that with sufficient mass loss and mixing, the products of previous $\mathrm{H}$ burning or current He-burning in the core would be exposed on the surface. Several different pathways, or "channels" by which a massive star could evolve to show W-R characteristics were proposed. Most participants, if polled, would have agreed with Lindsey Smith's remark, quoted above, from her summary talk. The concept of WO types as a separate class was introduced. Extensive IUE data and high resolution spectra were presented and analyzed. The first X-ray data were given; near infrared spectrophotometry was provided. The atmospheric models, while still "single point" approximations, were able to predict with some accuracy a few of the lines observed from the UV to the near IR. Considerable progress in binary star orbit analysis was given, with particular attention to the masses of W-R stars and their companions. The concept of and evidence for compact companions in certain W-R systems was argued about at length. The interaction of W-R stars with their environments, in the form of "rings", was offered and some of the first evidence of W-R stars beyond the Magellanic Clouds was shown.

As our Chairman has remarked in his opening address, the number of published papers on W-R stars has increased steadily in the past two decades. At our meeting here, the topics seem to have fallen into eight main categories as follows: i) Standard Atmospheric Models, Composition, Mass loss [20]; ii) Variability, Instabilities [10]; iii) Binaries [16]; iv) Environments [11]; v) Evolution, Luminous Blue Variables [16]; vi) W-R Descendants, SNIb [6]; vii) W-R in Other Galaxies, Initial Composition [11]; viii) W-R in Giant HII regions, W-R Galaxies [7]; unclassified [5]. The numbers following each topic in brackets are "weighted" percentages of the number of scheduled papers: the weighting is 3 times the number of oral papers plus the number of poster papers as listed in the preliminary program. These numbers can be used to give one a feeling for the emphasis in each area at this conference but should not be taken more seriously than that.

I will now consider each of these areas in turn and give my personal opinion of the highlights of the work as reported here, both by the oral reviews and contributions and by the poster papers. 


\section{i) Standard Atmospheric Models, Composition, Mass Loss}

Finally, and after much work by several groups, it has become possible to follow the sage advice of Ann Underhill in 1968 (as quoted in the beginning of this talk) and derive STANDARD ATMOSPHERIC MODELS which solve self-consistently the radiative transfer and statistical equilibrium equations throughout a moving extended atmosphere. These models assume a pure helium composition for the opacity, spherical symmetry, a monotonic velocity law, time independence and homogeneity. I shall return to these assumptions below but I cannot stress strongly enough that these models, constructed independently by several groups, are able to reproduce the observed continuum over several decades of wavelength and the strength and shape of most emission lines to better than a factor 2 . In my view this is the major theoretical advance in the study of W-R stars at this meeting.

Not yet included in the models are line blanketing, particularly the $\mathrm{FeV}$ and FeVI emission line blends below $1500 \AA$ for which there is now direct evidence. Furthermore, the velocity law adopted was arbitrary (but similar for all models) and has not been derived from first principles. To do so is an important next step that will involve hydrodynamical calculations which may be amenable to treatment in the next decade with the advent of very powerful computers.

In addition to fitting the observed spectrum, the standard atmospheric models give predictions which can be compared with other evidence. For WN stars, the mass loss rates from the line spectra are found to be in the range $10^{-4}$ to $10^{-5}$ solar masses/yr, similar to that obtained from the radio free-free estimates. The composition is clearly anomalous with respect to solar values and is in quantitative accord with that predicted by STANDARD EVOLUTION MODELS for massive stars which include mass loss and mixing. The Teff inferred from the models seems to be a little cool for the WNL stars with hydrogen, and some WNE stars, compared to evolution predictions; for the hottest WNE stars the Teff appear to be consistent with such predictions. The luminosities, $L$, inferred from the standard atmospheric models are nearly all too faint compared to the predictions of the standard evolution models. This has led several investigators to propose that the mass loss rates of W-R stars are proportional to some power of the mass (greater than unity) which is also indicated by the empirical relationship between these parameters. Similarly, the observed mass of many WN stars seems small compared to that expected from evolution of massive stars, unless a mass dependent mass loss rate for W-R stars is adopted.

It appears that in WN stars nitrogen blanketing will not play a major role in the structure of the atmosphere. In WC stars, the standard atmospheric models will need to include the effects of carbon as an opacity source before any meaningful comparisons to luminosity and Teff are made. The mass loss rates and compositions are consistent, as for the WN sequence, with the radio free-free emission, and the predictions of the evolution models, respectively.

Could W-R stars have significant departures from spherical symmetry? This theme came up at several points in our proceedings and I would like to personally address this issue. In the one case (HD 50896) where we are fairly certain of the rotational origin of the variable polarization, the period is 3.7 days and the equatorial velocity relatively low and far from a "critical value". The flat topped profiles of 5696 CIII (and other lines) in WC stars sets an upper limit on departures from sphericity as such a line can only be produced in a spherical shell. I have not seen any quantitative discussion of this straightforward and very model independent observation. Many W-R stars show no changes in polarization with time. There is no evidence of non-sphericity in eclipsing binary systems. All of these points together suggest to me that spherical symmetry is a pretty good approximation for most, if not all, W-R stars. 
Could W-R stars have substantial non-homogeneities and/or a non-monotonic velocity law? Here the evidence pro or con is sketchy and clearly needs more work. As I will discuss presently, there is clear evidence for some inhomogeneities in the winds of W-R stars. The extent to which this controls, or is dominated by, the wind is currently unsettled.

A new concept which has recently emerged in the study of winds of W-R (and OB type) stars is that of a distinction between the "edge" velocity of the P Cygni profile, and the "terminal" velocity. The latter is a fundamental parameter of the wind and is needed to connect the radio emission measures to the mass loss rates. It appears that this velocity is best estimated from the violet most part of the "zero continuum (or black)" absorption profile; the edge velocity, typically about $25 \%$ larger, is the maximum violet displacement of any portion of the absorption profile. The difference may be due to low density higher velocity material coming from "reverse shocks" in the stellar winds of W-R (and OB type) stars. The realization of this velocity effect lowered previously determined radio mass loss rates by $25 \%$; on the other hand, the recognition that the ionization balance in the outer part of the wind was lower raised previous radio mass loss rates by a factor 2 . In any case, the mass loss rate predictions of the standard atmospheric models, and the observed free-free measurements are now in good agreement.

Are W-R winds driven entirely by radiation pressure? While there are those who advocate this, and I count myself among them, there is a problem for a few W-R stars with required multiple scatterings for a given photon of 10 to 40 or more. A consensus among some theorists is that multiple scatterings of up to 10 can reasonably be expected in a radiatively driven wind but larger numbers are not possible. It could be that in those few stars with larger inferred multiple scattering values the $\mathrm{L}$ has been underestimated due to an inadequate bolometric correction. This will have to be sorted out in the future.

ii) Variability, Instabilities

There has been considerable progress in observations of the variability of W-R stars involving measurements in the continuum, the emission lines and in polarimetry. Extrinsic variability is produced in close binary systems, due mostly to geometric distortion effects which are reasonably well understood. Of particular value are polarimetric measurements which enable one to solve for the orbital inclination and thus better estimate the stellar masses in well studied orbital systems. Many W-R stars have intrinsic variability in the emission lines at the few percent level; the WN8 and WC9 stars have somewhat larger amplitude changes which has been interpreted as being due to their larger radii and more sluggish winds. A few W-R stars appear to be constant over time in their spectral features.

The cause of such variability would seem to be instabilities in the radiatively driven winds, which are predicted to be present. The theory suggests the presence of "reverse shocks" which lead to low densities at high velocities and high densities at low velocities in the winds. This is exactly what is needed to account for the distinction between the "edge" and "terminal" velocities observed in the $P$ Cygni profiles of certain ions. I would expect considerable quantitative advances in such dynamical wind problems in the next few years.

Radial pulsation has been predicted by some investigators but has not yet been demonstrated to be present from observations. Non-radial pulsations, predicted by other investigators, may have been found in a few cases but such an interpretation is currently controversial and this issue is not settled. 
iii) Binaries

The study of binary systems with $\mathrm{O}$ type companions continues. Eclipse effects can be used to probe the wind structure of the W-R component: e.g., the establishment of the FeV, FeVI pseudo-continua. A summary of the properties of the known $W-R$ binaries has provided valuable information on the individual masses and the mass ratios. While there may be one established W-R plus W-R binary, the evidence is not compelling and now, in contrast to a decade ago, the double star scenarios suggest none should be found. A detailed analysis of an eccentric orbit eclipsing binary system in the SMC and useful estimation of its parameters needs to be noted.

There now seems to be little evidence for W-R systems with compact companions: neutron stars or black holes, although one or two objects remain as possible candidates.

\section{iv) Environments}

A major new result was the findings of nebular 4686 HeII surrounding a few early type W-R stars. A straightforward interpretation of the presence of this line requires equivalent Teff of 70000 or more. A large b.c. would be inferred for such stars, thus possibly easing the multiple scattering limit problem. W-R "rings" can be used as probes of the properties of the exciting W-R stars and they give Teff and compositions consistent with the predictions of the standard atmospheric models.

There was evidence presented for bi-polar outflow in those few cases where there is an observed asymmetric nebulosity. How non-spherical is the ejecting W-R star in such a case? I was disappointed not to have seen this discussed. Massive stars, along with their local and giant HII region environments, produce copious amounts of high energy photons. Surface magnetic fields of few gauss fields might help understand the X-rays observed in $\mathrm{W}-\mathrm{R}$ winds. There will be a major observational impact on the high energy astrophysics of W-R stars in the next decade with the launch of several new satellites.

\section{v) Evolution, Luminous Blue Variables}

It appears from the standard evolution models that mass loss and mixing in massive stars can produce helium rich objects which have masses, luminosities and compositions much like the observed W-R stars. In the current theory, they have a singular dependence on the "metal abundance", $z$. It would be expected from these models that as $\mathrm{z}$ goes down, the lower mass limit above which a single W-R star can be formed will go up. This would affect the W-R/O star ratio. Furthermore, the number ratio of WN/WC subtypes should also be a simple function of $\mathrm{z}$, getting larger as $\mathrm{z}$ decreases. Both effects come about because of the dependence of mass loss rate on $z$ and the resultant length of time a massive star will spend in each stripped, or "bare core" stage (H-burning products:WN, or He-burning products:WC).

The observed LBV may also play an important role in massive star evolution. Their major mass loss episodes and an estimated frequency of such occurrences, along with a steady outflow of material in between such events, make them prime candidates for being an important link between Of and W-R phases. An important and unresolved question is whether or not all W-R stars go through an LBV phase; some would appear to do so. Currently, the origin of the LBV phase is thought to be related to the Eddington limit which a massive star may approach at certain times in its evolution. LBV phases have not been included specifically in the standard evolution models but their effect would be similar to raising the effective mass loss rates during core H-burning. 
Massive stars that enter a Wolf-Rayet phase will all end up near 10 solar masses, irrespective of their initial masses, given a mass loss rate depending on a power of the mass as proposed by several investigators. This channeling down to a more or less uniform mass is an important prediction of the standard evolution models. It does not appear to be at odds with our current knowledge of W-R star masses which, however, all come from well studied binary systems in which the evolution may have proceeded differently.

There seems to be general assent among those modeling W-R star evolution that the "metal abundance $-z^{n}$ must play an important role. It is clear that this parameter will influence the mass loss rates in the stellar winds; its function in internal mixing is less settled. There is fairly strong evidence, at least from the solar vicinity and the Magellanic Clouds, that the value of " $\mathrm{z}$ " may have had a substantial influence on the numbers of WN and WC star produced. Recently, new values of the composition of the Magellanic Clouds has been reported by Dopita (private communication at this meeting; see also the papers in the literature). The new numbers are as follows (they have not been incorporated in the papers presented at this conference):

$\begin{array}{cccc} & \mathrm{O} / \mathrm{H} & \mathrm{Fe} / \mathrm{H} & \mathrm{WN} / \mathrm{WC} \\ \text { Solar } & 1 & 1 & 1 \\ \text { LMC } & 1 / 3 & 1 / 2 & 4 \\ \text { SMC } & 1 / 6 & 1 / 4 & 7:\end{array}$

The values of the $\mathrm{O} / \mathrm{H}$ and $\mathrm{Fe} / \mathrm{H}$ ratios in the $\mathrm{LMC}$ and SMC come from detailed analyses of supergiants, HII regions, planetary nebulae and $\mathrm{SN}$ remnants and are consistent with one another. What bothers me about this table is the extreme sensitivity of the WN/WC ratio to the metal abundance: the ratio changes from the solar vicinity to the LMC by a factor 4 , twice the difference in the $\mathrm{Fe} / \mathrm{H}$ ratio (which should control the stellar wind mass loss rate) and larger than that of the $\mathrm{O} / \mathrm{H}$ ratio (which controls the stellar structure equations). If we take such an interpretation at face value, we could turn the problem around and by simply obtaining the WN/WC ratio in other galaxies, estimate their " $\mathrm{z}$ " in a very straightforward and unambiguous manner. This seems just a little too simple for me (see also below).

\section{vi) W-R Descendants}

This topic appears for the first time at this meeting. There is a newly recognized class of SN called type Ib, (and Ic) in which the lines of hydrogen are absent, yet they appear, from their galaxy type origin and locations to be associated with massive progenitor stars (rather than low mass stars as for the SN1a). A number of investigators have suggested that such events might be derived from W-R stars which are well known for having lost their hydrogen. However, the SNIb frequency seems to be a little too high to be due to stars with initial masses as large as those proposed for W-R objects. It could be that some W-R binaries might end up as SNIb, but not all SNIB could be derived from W-R progenitors with the current statistics.

From a theoretical point of view, it is possible to carry a single 60 solar mass star through its various mass loss stages to arrive at catastrophic collapse phase which has properties much like those observed for SNIb. Furthermore, if single W-R stars do not become, SNIb, what is their fate? The issue of the W-R descendants is clearly not a settled one and much new observational and theoretical work is to be expected in the future. 
vii) W-R in Other Galaxies; Initial Composition

In a deep search in a direction down the Carina Arm some 13 additional W-R stars have been found. This suggests an incompleteness of about $25 \%$ in the counts of W-R stars near the solar vicinity. The impact on the total numbers estimated for the Galaxy is potentially important.

There were new discoveries of W-R stars in M33 and M31. There now appears to be little or no gradient in the WN/WC ratio with galactocentric distance in M33 and the value is near unity in M31 (which has a higher than solar value of $\mathrm{z}$ ). The statistics are seemingly secure given the numbers of stars involved. Thus the inferred WN/WC ratios did not appear to follow the simple prescription of dependence on $\mathrm{z}$ as suggested by the relations for the LMC and SMC. What other factors might influence the WN/WC ratio? This will need to be sorted out by additional observations of other galaxies and detailed examinations of completeness of the stellar samples so far considered.

viii) W-R in Giant HII regions, W-R Galaxies

Additional W-R stars have been identified in several of the Giant HII regions of M33. In these recent star forming regions, the WN/WC ratio is large! Clearly these do not follow the simple $\mathrm{z}$ dependence predicted by the standard evolution models. Could it be that the relatively bright WN stars in such HII regions are in their H-burning stages, thus lasting a relatively long time? Newly discovered W-R stars in several other nearby galaxies were reported and the search procedures were reviewed.

Wolf-Rayet galaxies are those in which a broadened $4686 \mathrm{HeII}$ emission line has been detected in the integrated spectrum. Some few dozen are known, typically as blue compact galaxies, but with relatively heterogeneous properties of luminosity and composition. In some cases, the W-R stars seem to appear in bright "knots", or supergiant HII regions, within star forming galaxies. An unanswered question is why W-R stars appear in some, but certainly not all, galaxies with other evidence of recent star forming episodes. Population synthesis models of $17 \mathrm{~W}-\mathrm{R}$ galaxies suggested ages of 3-6x10 years, implying a relatively recent "starburst". The evidence seems to favor a relatively "flat" IMF, compared to the solar vicinity, with no dependence of the slope on $\mathrm{z}$ for the studied systems.

This concludes my summary of the papers presented at the meeting. I would like to close by making a list of several issues which have engaged much of our attention in the past but now should be considered as "settled", and then addressing where I think the future decade of research in W-R stars will be leading.

\section{Settled Issues}

W-R stars represent a normal ending point of massive star evolution. WN, WC, and WO subtypes have anomalous abundances which represent the expected nuclear burning products in stars whose outer hydrogen rich material has been stripped away.

Massive star evolution models which include mass loss, mixing, and binary interactions can produce, in various combinations of importance, objects with properties similar to W-R stars.

Observed W-R continua and spectra can be modeled by a hot core - dense stellar wind: the standard atmospheric model. 


\section{Future decade - major themes}

Second generation models will need to be constructed which will combine the standard evolution model with a dynamical treatment of the stellar wind. These will need to account for the observed instabilities of W-R stars.

As extreme Population I objects, W-R stars should be excellent indicators of galactic structure. Where are the spiral arms of our Galaxy? Does our Galaxy have 2, or 3, major features? Is it an Sb or Sc type, or something in between?

Extensive population studies of individual W-R stars in more and more distant galaxies will be undertaken. The total numbers compared to the parent $O$ star population and the WN/WC ratios will be used to examine the predicted simple dependence on $\mathrm{z}$. It may be possible to investigate the environments of these galaxies from their massive star populations. Certainly the recent massive star formation rate can be evaluated.

W-R stars will be used to investigate "starburst" phenomena in even more distant galaxies where only the integrated spectrum is available. Is the IMF different in such environments?

"Primeval" blue galaxies may now have been detected at redshifts of about 1. One would expect that in such galaxies the initial massive stars that produced much of our current "metals" would be present. Are W-R stars also evident? Have they played a major role in the initial composition changes of our universe?

In closing, I see many opportunities for research on W-R stars, both those that are relatively nearby that can be studied in detail, and those that are at very distant locations that can only be observed in ensemble. In particular, now that they are better understood as evolved massive stars, they will be guideposts to our comprehension of star formation and galaxy evolution itself.

\section{REFERENCES}

Bappu, M.K.V. and Sahade, J. 1973 Wolf-Rayet and High-Temperature Stars, IAU Symposium \#49 (D. Reidel, Dordrecht)

De Loore, C.W.H. and Willis, A.J. 1982 Wolf-Rayet Stars: Observations, Physics, Evolution, IAU Symposium \#99 (D. Reidel, Dordrecht)

Gebbie, K.B. and Thomas, R.N. 1968 Wolf-Rayet Stars Proceedings of a Symposium, NBS Special Publication 307 (U.S. Government Printing Office, Washington D.C.)

Smith, L.F. 1982 in Wolf-Rayet Stars: Observations, Physics, Evolution, eds. C.W.H. De Loore and A.J. Willis (D. Reidel, Dordrecht), p. 599

Underhill, A.B. 1968 in Wolf- Rayet Stars, eds. K.B. Gebbie and R.N. Thomas (U.S. Government Printing Office, Washington D.C.), p. 185 